\title{
Formation and migration of vacancy defects in GeSe and SnSe
}

\author{
S. Zhang ${ }^{\mathrm{a}, \mathrm{b}}$, M.L. Li, ${ }^{\mathrm{a}}$ M. Jiang ${ }^{\mathrm{a}}$, H.Y. Xiao ${ }^{\mathrm{a},{ }^{,}, \text {David O. Scanlon }}{ }^{\mathrm{b}, \mathrm{c}, \mathrm{d}}$, X.T. Zu ${ }^{\mathrm{a}}$ \\ ${ }^{a}$ School of Physics, University of Electronic Science and Technology of China, Chengdu \\ 610054, China \\ ${ }^{b}$ Department of Chemistry, University College London, 20 Gordon Street, London \\ WC1H 0AJ, UK \\ ${ }^{c}$ Thomas Young Centre, University College London, Gower Street, London WC1E 6BT, \\ $U K$ \\ ${ }^{d}$ Diamond Light Source Ltd., Diamond House, Harwell Science and Innovation Campus, \\ Didcot, Oxfordshire OX11 ODE, United Kingdom
}

\begin{abstract}
GeSe and SnSe have great potential in nuclear detector devices. Under irradiation, the formation and migration of point defects may affect their properties and performance significantly. In this study, a comparative study of vacancy formation and migration in GeSe and $\mathrm{SnSe}$ has been carried out using first-principles methods. It is shown that in both compounds cation vacancies form much easier than anion vacancies, and that cation vacancies have a lower barrier to migration than anion vacancies in GeSe and SnSe. For both Ge vacancy and Sn vacancy, the migration is anisotropic and the [322] direction is the most favorable migration pathway. The migration energy barrier are $0.54 \mathrm{eV}$ for Ge vacancy and $0.46 \sim 0.52 \mathrm{eV}$ for Sn vacancy, suggesting that vacancy clusters are relatively easy to form in both compounds, which may influence
\end{abstract}


the application of GeSe and SnSe in nuclear detector devices.

Keywords: GeSe; SnSe; Defect formation; Defect migration; Density functional theory

* Corresponding author. E-mail address: hyxiao@uestc.edu.cn (H.Y. Xiao) 


\section{Introduction}

Selenium-based layered compounds $\mathrm{XSe}(\mathrm{X}=\mathrm{Ge}, \mathrm{Sn})$, in which the $\mathrm{X}$-Se bilayers are attached by the van der Waals force and weak bonding [1], are IV-VI compounds with nontoxic and environmentally friendly characteristics among the selenides $[2,3]$. In recent years, the GeSe and SnSe compounds have attracted extensive attention, due to their large band gap [4], anisotropic spin transport properties [1], high mobility [5], high stability [6], and high energy resolution [7], which are beneficial for their potential applications in solar cells [8,9], optoelectronic devices [10,11], thermoelectric materials $[12,13]$, electronic memory devices [14], and lithium intercalation batteries $[15,16]$. Especially, the GeSe $[17,18]$ and $\mathrm{SnSe}[19,20]$ have been reported to be promising candidates as nuclear detector devices $[20,21]$.

The radiation detection devices are frequently exposed to different radiation environments during their applications, e.g., neutron, electron and gamma radiation, which may result in the generation of various defects like vacancies interstitials, and antisite defects, and these defects influence the performances of detector devices [2224]. Investigation of the defect formation and migration, is therefore important to gain fundamental understanding of the material properties under radiation environment. The defect properties of $\mathrm{GeSe}$ and $\mathrm{SnSe}$ have attracted the attention of researchers since $2010[25,26]$. For GeSe, it has been found that the Ge vacancy $\left(\mathrm{V}_{\mathrm{Ge}}\right)$ possesses the lowest formation energy under both Ge-rich and Se-rich growth conditions [18] and it has been postulated $\mathrm{V}_{\mathrm{Ge}}$ results in insulating-to-metallic transition in the twodimensional material [27]. For SnSe, the Sn vacancy $\left(\mathrm{V}_{\mathrm{Sn}}\right)$ and Se vacancy $\left(\mathrm{V}_{\mathrm{Se}}\right)$ are 
easily formed under Se-rich and Sn-rich conditions, respectively [28]. Huang et al. also found that the type of created defects depends on the chemical environment, and the $\mathrm{V}_{\mathrm{Sn}}$ and $\mathrm{Sn}$ interstitial defects result in metallic properties of $\mathrm{SnSe}$ [29]. Experimentally, Straitrova et al. suggested that the $\mathrm{V}_{\mathrm{Sn}}$ dominates in the low-temperature region, and $\mathrm{V}_{\mathrm{Se}}$ and vacancy clusters will form in the high-temperature region [30]. Despite these investigations, the defect migration behavior of GeSe and SnSe still remains unknown. Under irradiation defect migration can affect directly the number, distribution and agglomeration of defects and consequently microstructure and physical properties, which may influence the overall performance of nuclear radiation detection device. Investigation of migration behavior of GeSe and $\mathrm{SnSe}$, thus, is necessary to understand their radiation response.

In the present work, a density functional theory (DFT) method is employed to investigate vacancy formation and migration in $\mathrm{XSe}(\mathrm{X}=\mathrm{Ge}, \mathrm{Sn})$. The vacancy formation energy, migration energy barrier and migration pathway in $\mathrm{XSe}(\mathrm{X}=\mathrm{Ge}, \mathrm{Sn})$ are determined and compared. Besides, the effects of vacancy defect on electronic structures of $\mathrm{XSe}(\mathrm{X}=\mathrm{Ge}, \mathrm{Sn})$ are studied. The presented results may advance the fundamental understanding of the behavior of GeSe and SnSe under irradiation.

\section{Computational details}

All the calculations are carried out using the Vienna Ab-initio Simulation Package (VASP) code based on the projector augmented wave method $[31,32]$. The exchangecorrelation effects are treated using the Perdew-Burke-Ernzerhof functional under the 
generalized gradient approximation (GGA) [33]. A cutoff energy of $500 \mathrm{eV}$ for plane wave basis set and a $4 \times 4 \times 4$ Monkhorst-Pack k-mesh for Brillouin-zone integrations are used for structural relaxation and electronic structure calculations. The convergence criteria for total energies and forces are $10^{-4} \mathrm{eV}$ and $10^{-3} \mathrm{eV} / \AA$, respectively. The full relaxation is performed during the structural optimization. For defective GeSe and $\mathrm{SnSe}$, a $1 \times 3 \times 3$ supercell consisting of 72 atoms is employed to avoid the interaction between the adjacent defects. In the present work, four types of vacancy, i.e., Se vacancy $\left(\mathrm{V}_{\mathrm{Se}}\right)$ and Ge vacancy $\left(\mathrm{V}_{\mathrm{Ge}}\right)$ in GeSe, and $\mathrm{V}_{\mathrm{Se}}$ and $\mathrm{Sn}$ vacancy $\left(\mathrm{V}_{\mathrm{Sn}}\right)$ in $\mathrm{SnSe}$, are taken into account, as shown in Figure 1. The climbing image nudge elastic band (CI-NEB) [34,35] theory is used to find the minimum energy pathway (MEP) for vacancy diffusion. Besides, the transition state configuration at the saddle point is provided. The considered migration path for vacancy defects are illustrated in Figure 1.

\section{Results and discussion}

\subsection{The ground state properties of bulk GeSe and SnSe}

The GeSe and SnSe belong to the family of compounds with layer-type orthorhombic structure in the $D_{2 h}^{16}$ (no. 62) space symmetry group at low temperature, which can be considered as a distorted $\mathrm{NaCl}$ rocksalt structure [36]. The unit cell includes eight atoms, and the atoms of $X(X=G e, S n)$ and $\mathrm{Se}$ are bonded by three Se and $X(X=G e, S n)$ atoms, respectively, as shown in Figure 1. It is known that van der Waals force exists in the layer-type materials, whereas the X-Se bilayers are also attached by the weak bonding. 
electron of Sn and much stronger than that of the van der Waals interaction. In addition, the van der Waals corrections high-estimates the binding energy and can not give the better lattice structure than that of PBE [37], while the van der Waals corrections will result in the expensive calculations. Thereby, the van der Waals corrections not considered in present work. Table 1 presents the optimized lattice constants, along with the experimental and other theoretical results for comparison. For GeSe, the obtained lattice parameters are $a=11.12 \AA, b=3.87 \AA$ and $c=4.55 \AA$, which are larger than the experimental values of $a=10.84 \AA, b=3.83 \AA$ and $c=4.39 \AA$ [38] due to the overestimation of GGA method. In the literature, the calculated lattice constants range from 10.85 to $11.31 \AA, 3.83$ to $3.91 \AA, 4.33$ to $4.49 \AA$ for $a, b$ and $c$, respectively [3941]. For SnSe, the calculated lattice constants are $a=11.74 \AA, b=4.21 \AA$ and $c=4.54 \AA$, which are in reasonable agreement with experimental data of $a=11.50 \AA, b=4.15 \AA$ and $c=4.44 \AA[42]$ and other theoretical data $(a \sim 11.53-11.81 \AA, b \sim 4.17-4.22 \AA$ and $c \sim 4.4-$ $4.57 \AA$ ) [39-41]. The band gaps of GeSe and SnSe are determined to be 0.84 and 0.61 $\mathrm{eV}$, respectively, which are comparable to the calculated values of 0.87 and $0.69 \mathrm{eV}$ reported by Ding et al [39].

\subsection{Formation energy of vacancies in GeSe and SnSe}

The vacancy (absence of an atom) is one of common intrinsic defects that is of central importance to many physical and chemical processes taking place in compounds. The formation energies of vacancy defects under both Se-rich and Ge-rich/Sn-rich conditions are presented in Table 2, along with other calculated results for comparison. 
The vacancy formation energy can be calculated from the expression: $E_{f}=E_{\text {defect }}-$ $E_{\text {perfect }}+\sum_{i} \Delta n_{i} \mu_{i}[43,44]$. Here, $E_{\text {defect }}$ is the total energy of the defective supercell, $E_{\text {perfect }}$ is the total energy of the perfect supercell, $\Delta n_{i}$ is the number of atoms removed from the perfect cell and $\mu_{i}$ is the chemical potential of species $i$. For bulk $\mathrm{XSe}(\mathrm{X}=\mathrm{Ge}, \mathrm{Sn})$, the chemical potential of $\mathrm{X}$ and Se satisfy the following constraints: $\mu_{X} \leq \mu_{X}^{\text {bulk }}, \mu_{S e} \leq \mu_{S e}^{\text {bulk }}$ and $\mu_{X}+\mu_{S e}=\mu_{X S e}^{\text {bulk }}$ [45], where $\mu_{X}^{\text {bulk }}$, $\mu_{S e}^{\text {bulk }}$ and $\mu_{X S e}^{\text {bulk }}$ are the total energy of bulk X, bulk Se and bulk XSe, respectively. Under X-rich and Se-rich growth conditions, the chemical potential of Se and X can be defined by: $\mu_{X}=\mu_{X}^{\text {bulk }}, \mu_{S e}=\mu_{X S e}^{\text {bulk }}-\mu_{X}$ and $\mu_{S e}=\mu_{S e}^{\text {bulk }}, \mu_{X}=\mu_{X S e}^{\text {bulk }}-\mu_{S e}$, respectively. As shown in Table 2, for GeSe under Ge-rich condition, the formation energies of $1.33 \mathrm{eV}$ for $\mathrm{V}_{\mathrm{Ge}}$ and $1.59 \mathrm{eV}$ for $\mathrm{V}_{\mathrm{Se}}$ compare well with the corresponding values of 1.14 and $1.59 \mathrm{eV}$ reported by Mukherjee et al. [18]. Under Se-rich condition, the formation energies of $0.86 \mathrm{eV}$ for $\mathrm{V}_{\mathrm{Ge}}$ and $2.06 \mathrm{eV}$ for $\mathrm{V}_{\mathrm{Se}}$ are also comparable to the results of Mukherjee et al. [18]. It is noted that the formation energy of $\mathrm{V}_{\mathrm{Ge}}$ is smaller than that of $\mathrm{V}_{\mathrm{Se}}$ under both Se-rich and Ge-rich growth conditions, indicating that $\mathrm{V}_{\mathrm{Ge}}$ is more favorable in GeSe crystal. Similar phenomenon has been observed by Gomes et al. [27], who carried out DFT/GGA calculations on GeSe, and found the $\mathrm{V}_{\mathrm{Ge}}$ has smaller formation energy than $\mathrm{V}_{\mathrm{Se}}$ under both Se-rich and Ge-rich conditions. For SnSe, the formation energy of $1.74 \mathrm{eV}$ for $\mathrm{V}_{\mathrm{Sn}}$ is larger than that of $1.23 \mathrm{eV}$ for $\mathrm{V}_{\mathrm{Se}}$ under $\mathrm{Sn}$ rich condition, and the formation energy of $0.84 \mathrm{eV}$ for $\mathrm{V}_{\mathrm{Sn}}$ is smaller than that of 2.02 $\mathrm{eV}$ for $\mathrm{V}_{\mathrm{Se}}$ under Se-rich condition, suggesting that the vacancy formation in SnSe crystal depends on the chemical environment, which is consistent with the results 
reported by Zhou et al. [28]. These results show that in both compounds the cation vacancy is much easier to form than anion vacancy under Se-rich condition, and the $\mathrm{V}_{\mathrm{Ge}}$ and $\mathrm{V}_{\mathrm{Sn}}$ have comparable formation energies. It is also noted that under cation-rich condition the $\mathrm{V}_{\mathrm{Ge}}$ and $\mathrm{V}_{\mathrm{Se}}$ prefer to form in GeSe and SnSe, respectively, and such discrepancy may be resulted from the difference in interaction between $<$ Se-Ge $>$ and $<$ Se-Sn $>$ bonding.

\subsection{Migration pathway and energy barrier of vacancy defects in GeSe and SnSe}

\subsubsection{Migration of cation vacancy in GeSe and $\mathrm{SnSe}$}

Using NEB method, the $\mathrm{V}_{\mathrm{Ge}}$ and $\mathrm{V}_{\mathrm{Sn}}$ vacancy migration in GeSe and SnSe are further investigated. The migration barriers for $\mathrm{V}_{\mathrm{Ge}}$ in GeSe and $\mathrm{V}_{\mathrm{Sn}}$ in $\mathrm{SnSe}$ along the [010], [001], [322] and [011] directions (as shown in Figure 1) are plotted in Figures 2 and 3, respectively. For $\mathrm{V}_{\mathrm{Ge}}$ migration along the [010] direction, there are two energy barriers, i.e., 0.78 and $0.14 \mathrm{eV}$, to overcome along the minimum energy path. It is noted that the two saddle-points has almost identical total energies, which is due to the symmetrical geometrical structure along the [010] direction originating from the orthorhombic structure of GeSe. In the case of $\mathrm{V}_{\mathrm{Ge}}$ migration along the [322] direction, there are two different saddle-points with the same energy barriers $(0.54 \mathrm{eV})$, and an energetically stable state is found between two saddle-points. As shown in Figure 2, the migration of $\mathrm{V}_{\mathrm{Ge}}$ along the [010] direction is impeded by the energy barriers of 0.87 and $0.29 \mathrm{eV}$. It is noticable that the $\mathrm{V}_{\mathrm{Ge}}$ defect is more difficult to migrate along the [011] direction, as indicated by the large energy barriers of 1.30 and $0.61 \mathrm{eV}$. Obviously, the energy 
barriers of $\mathrm{V}_{\mathrm{Ge}}$ strongly depend on the migration directions, and the $\mathrm{V}_{\mathrm{Ge}}$ defect prefers to migrate along the [322] direction.

As shown in Figure 3, the migration behavior of $V_{S n}$ is also anisotropic. The $V_{S n}$ defect is easier to migrate along the [322] direction, as indicated by the relatively small energy barrier values of $0.46 \sim 0.52 \mathrm{eV}$. The vacancy diffusion along the [011] direction is the most difficult due to the large energy barrier of $2.02 \mathrm{eV}$. This is similar to the case of $\mathrm{V}_{\mathrm{Ge}}$ in GeSe. For $\mathrm{V}_{\mathrm{Sn}}$ migration along the [010] direction, the migration energy barriers are 0.76 and $0.10 \mathrm{eV}$, and the total energy difference between the two saddlepoints is only $0.02 \mathrm{eV}$. The migration energy barriers along the [001] direction are comparable to those for [010] direction, i.e., 0.72 and $0.23 \mathrm{eV}$, in which the $\mathrm{V}_{\mathrm{Sn}}$ moves from one lattice site to its nearest-neighboring lattice site. As shown in Figures 2 and 3, the migration mechanisms for $\mathrm{V}_{\mathrm{Ge}}$ along the [010] and [001] directions exhibit similar character to those for $\mathrm{V}_{\mathrm{Sn}}$, as indicated by the comparable migration barriers and similar migration paths including two saddle-points. It is noted that the migration barrier of $0.54 \mathrm{eV}$ for $\mathrm{V}_{\mathrm{Ge}}$ along the [322] direction is comparable to that of $0.46 \sim 0.52 \mathrm{eV}$ for $\mathrm{V}_{\mathrm{Sn}}$, indicating that both $\mathrm{V}_{\mathrm{Ge}}$ and $\mathrm{V}_{\mathrm{Sn}}$ are easily to diffuse along the [322] direction, and the vacancy cluster may form in GeSe and SnSe crystals. This is consistent with the experimental results reported by Straitrova et al. [30], who observed the formation of vacancy clusters in SnSe crystal.

To further understand the migration behavior of vacancy defects, the migration path and the atomic structures of saddle-point and local energy minimum site are analyzed. For the migration along the [322] direction, both $\mathrm{V}_{\mathrm{Ge}}$ and $\mathrm{V}_{\mathrm{Sn}}$ have two saddle-points 
(denoted as point A and B) and one local energy minimum site (as shown in Figure 2 and Figure 3). The local geometrical structures of saddle-point A, local minimum site and saddle-point $\mathrm{B}$ for $\mathrm{V}_{\mathrm{Ge}}$ and $\mathrm{V}_{\mathrm{Sn}}$ migration are shown in Figure 4 and related bonding distances are given in Table 3. For $\mathrm{V}_{\mathrm{Ge}}$ migration, at saddle-point $\mathrm{A}$ the $\mathrm{Ge}$ atom is coordinated by three Se atoms and its location is $0.46 \AA$ away from the plane consisting of Se1, Se2 and Se3 atoms [see Figure 4(a)]. The bonding distances between Ge and its three neighboring $\mathrm{Se}(\mathrm{Se} 1, \mathrm{Se} 2$ and $\mathrm{Se} 3)$ atoms in defective GeSe are determined to be 2.34, 2.51 and $2.48 \AA$, respectively, exhibiting asymmetric characters. As for the local minimum site, the Ge atom is located at the center of a tetrahedron and coordinated by four Se atoms. The $<\mathrm{Ge}-\mathrm{Se}>$ distances are calculated to be $2.36,2.42,2.53$ and $2.49 \AA$ for $\mathrm{Se} 1, \mathrm{Se} 2, \mathrm{Se} 3$ and $\mathrm{Se} 4$, respectively. When the Ge vacancy migrates further, the chemical bonding for Ge atom changes again. At saddle point $\mathrm{B}$, bond breakage for $<\mathrm{Ge}-\mathrm{Se} 3>$ occurs and the Ge atom is located at a position with a vertical distance of 0.3 $\AA$ from the plane consisting of $\mathrm{Se} 1, \mathrm{Se} 2$ and $\mathrm{Se} 4$ atoms. The $<\mathrm{Ge}-\mathrm{Se} 1>,<\mathrm{Ge}-\mathrm{Se} 2>$ and $<$ Ge-Se4 $>$ bonding distances are 2.30, 2.36 and $2.38 \AA$, respectively. As compared with the case of local energy minimum site, these bonds all become more covalent.

As shown in Figure 4(b), the local geometrical structures of saddle-point A, local minimum site and saddle-point $\mathrm{B}$ for $\mathrm{V}_{\mathrm{Sn}}$ migration show very similar character to those for $\mathrm{V}_{\mathrm{Ge}}$ migration. At saddle point $\mathrm{A}$, the $\mathrm{Sn}$ atom is three-coordinated with $\mathrm{Se} 1, \mathrm{Se} 2$ and $\mathrm{Se} 3$ atoms with bonding distances of $2.53,2.61$ and $2.66 \AA$, respectively, and the vertical distance between $\mathrm{Sn}$ and the (Se1-Se2-Se3) plane is $0.40 \AA$. In the case of local energy minimum site, the Sn atom migrates through a tetrahedron consisting of Se1, 
$\mathrm{Se} 2, \mathrm{Se} 3$ and $\mathrm{Se} 4$ atoms, and the respective $<\mathrm{Sn}-\mathrm{Se}>$ bonding distances are 2.54, 2.56, 2.70 and $2.68 \AA$. At saddle point $\mathrm{B}$, the $<\mathrm{Sn}-\mathrm{Se} 3>$ bond breaks and the $\mathrm{Sn}$ atom is coordinated with $\mathrm{Se} 1, \mathrm{Se} 2$, and $\mathrm{Se} 4$ atoms with bonding distances of 2.61, 2.57 and 2.66 $\AA$, respectively. The vertical distance between $\mathrm{Sn}$ and the (Se1-Se2-Se4) plane is calculated to be $0.4 \AA$. Comparing the $<\mathrm{Ge}-\mathrm{Se}>$ and $<\mathrm{Sn}$-Se $>$ distances in the local geometrical structures, we find that the differences in bonding distances between $<\mathrm{Ge}$ $\mathrm{Se}>$ and $<\mathrm{Sn}-\mathrm{Se}>$ bonds range from 0.1 to $0.31 \AA$, i.e., the $<\mathrm{Sn}-\mathrm{Se}>$ bonds are generally weaker than $<\mathrm{Ge}-\mathrm{Se}>$ bonds. In the literature, the ionic radii for $\mathrm{Ge}$ and $\mathrm{Sn}$ have been reported to be 1.21 and $1.40 \AA$, respectively [46]. Due to the larger ionic radii and lower electronegativity of $\mathrm{Sn}$, the $<\mathrm{Sn}-\mathrm{Se}>$ bonds are generally weaker than $<\mathrm{Ge}-\mathrm{Se}>$ bonds. Consequently, the $\mathrm{V}_{\mathrm{Sn}}$ migrates relatively easier than $\mathrm{V}_{\mathrm{Ge}}$ and vacancy clusters are easier to form in SnSe crystal.

\subsubsection{Migration of anion vacancy in GeSe and SnSe.}

For anion vacancy migration in GeSe and $\mathrm{SnSe}$, we also consider four migration directions, i.e., [010], [001], $[\overline{5} \overline{4} \overline{4}]$ and [011] directions (as shown in Figure 1). The results of migration barriers for $\mathrm{V}_{\mathrm{Se}}$ in GeSe and $\mathrm{SnSe}$ are plotted in Figures 5 and 6, respectively. For GeSe with $\mathrm{V}_{\mathrm{Se}}$ defect, as shown in Figure 5, the defect migration barrier along the [010] direction is $1.22 \mathrm{eV}$. Along the $[\overline{5} \overline{4} \overline{4}]$ direction, the $\mathrm{V}_{\mathrm{Ge}}$ migration is impeded by the energy barriers of $0.14 \mathrm{eV}, 0.21 \mathrm{eV}$ and $0.06 \mathrm{eV}$, which is the easiest migration direction. For $\mathrm{V}_{\mathrm{Se}}$ migration along the [001] and [011] direction, only one saddle-point exists and the migration energy barriers are 1.85 and $2.36 \mathrm{eV}$, 
respectively, which are larger than those along the [010] and $\overline{[5} \overline{4} \overline{4}]$ directions. For $\mathrm{SnSe}$ with $\mathrm{V}_{\mathrm{Se}}$ defect, the migration energy barrier is $1.83 \mathrm{eV}$ along the [010] direction, which is larger than the value of $1.07 \mathrm{eV}$ along the $[\overline{5} \overline{4} \overline{4}]$ direction and close to the value of $1.89 \mathrm{eV}$ along the [011] direction. In the case of [011] direction, the migration energy of $3.51 \mathrm{eV}$ is much larger than that along the other three directions, indicating that it is more difficult for $\mathrm{V}_{\mathrm{Se}}$ defect migration along this direction. It is noted that the migration energies of $\mathrm{V}_{\mathrm{Se}}$ in GeSe are larger than those of $\mathrm{V}_{\mathrm{Se}}$ in $\mathrm{SnSe}$ for each considered specific direction, i.e., $\mathrm{V}_{\mathrm{Se}}$ migration in $\mathrm{SnSe}$ is much easier to occur than that in GeSe. Besides, it is found that along each specific direction the migration energy of $\mathrm{V}_{\mathrm{Ge}}$ or $\mathrm{V}_{\mathrm{Sn}}$ is smaller than that of $\mathrm{V}_{\mathrm{Se}}$ in both GeSe and SnSe, suggesting that cation vacancy migration in XSe is easier to occur than anion vacancy migration.

\subsubsection{Migration of vacancies in GeSe and $\mathrm{SnSe}$ between two neighboring layers.}

The migration of cation and anion vacancies between two neighboring layers in GeSe and SnSe are also considered. The migration direction is indicated in Figure 1 and the energy change with diffusion coordinate is plotted Figure 7. It is noticeable that the vacancy migration in GeSe and $\mathrm{SnSe}$ between two neighboring layers are very similar to each other, and in all cases only one saddle-point exists along the migration path. In GeSe, the migration energy barrier of $1.33 \mathrm{eV}$ for $\mathrm{V}_{\mathrm{Ge}}$ is smaller than that of $1.86 \mathrm{eV}$ for $\mathrm{V}_{\mathrm{Se}}$. Similar phenomenon is also found in $\mathrm{SnSe}$, for which the migration energy barrier of $1.00 \mathrm{eV}$ for $\mathrm{V}_{\mathrm{Sn}}$ is smaller than that of $1.78 \mathrm{eV}$ for $\mathrm{V}_{\mathrm{Se}}$. These results indicate that cation vacancy migration between layers is easier than anion vacancy 
migration in both GeSe and SnSe.

\subsubsection{Effect of temperature on the migration energy barrier}

In order to investigate the effect of temperature on the migration barriers, the $\mathrm{V}_{\mathrm{Ge}}$ and $\mathrm{V}_{\text {Sn }}$ migration along the [322] direction are chosen for study due to easy formation of cations and lower migration energy. The structures of defective crystal are obtained based on the experimental the thermal expansion coefficients [47-49]. Figure 8 shows the variation of energy barriers of the $\mathrm{V}_{\mathrm{Ge}}$ and $\mathrm{V}_{\mathrm{Sn}}$ migration along the [322] direction with temperature. For the Ge vacancy in GeSe, the energy barrier decreases from 0.68 to $0.59 \mathrm{eV}$ when the temperature changes from 0 to $400 \mathrm{~K}$. Similar trend of migration barriers with temperature has been found in the case of $V_{S n}$ in $S n S e$, i.e., varying from 0.58 to $0.49 \mathrm{eV}$ as the temperature varies from 0 to $400 \mathrm{~K}$. These results show that the energy barriers decrease with increasing temperature, which has been observed in other materials like $\mathrm{Na}^{+}$ion migration barriers in $\mathrm{Na}_{11} \mathrm{Sn}_{2} \mathrm{PS}_{12}$ [50] and the oxygen and gallium vacancy migration barriers in $\beta-\mathrm{Ga}_{2} \mathrm{O}_{3}$ [51]. Additionally, we also found that the energy barrier values of $0.58 \sim 0.49 \mathrm{eV}$ for $\mathrm{V}_{\mathrm{Sn}}$ defect in $\mathrm{SnSe}$ are smaller than those for $\mathrm{V}_{\mathrm{Ge}}$ in $\mathrm{GeSe}$ along same direction, meaning that the cation vacancy in $\mathrm{SnSe}$ is easier to migrate under complex radiation environments.

\subsection{Effects of vacancies on the electronic structure of GeSe and SnSe}

The band structures of perfect and defective GeSe and $\mathrm{SnSe}$ are further calculated to explore the effects of vacancy defects on their electronic properties. As shown in Figure 
9(a) and (c), the band gap of $0.64 \mathrm{eV}$ for defective GeSe with $\mathrm{V}_{\mathrm{Se}}$ is found to be smaller than the value of $0.84 \mathrm{eV}$ for perfect $\mathrm{GeSe}$, while the introduction of $\mathrm{V}_{\mathrm{Ge}}$ results in an insulating-to-metallic transition. Similarly, the $\mathrm{V}_{\mathrm{Se}}$ defect in SnSe also results in a reduction of band gap from $0.61 \mathrm{eV}$ to $0.40 \mathrm{eV}$, and the $\mathrm{V}_{\mathrm{Sn}}$ defect also leads to a transition from insulator to metal. These results suggest that the introduction of vacancy defect in GeSe and SnSe modifies their band structures significantly.

The projected density of state (DOS) distribution for perfect and defective GeSe and SnSe are presented in Figure 10. For perfect GeSe, the valance band maximum (VBM) is dominated by Se $4 p$ states hybridized with Ge $4 p$ and Ge $4 s$ states, and the conduction band minimum (CBM) is mainly contributed by the $4 p$ states of Ge and Se. Comparing the DOS of defective and perfect GeSe, we find that the metallicity of $\mathrm{V}_{\mathrm{Ge}}$ originates from the interaction between $\mathrm{Se} 4 p, \mathrm{Ge} 4 p$ and Ge $4 s$ states, and the impurity energy level of $\mathrm{V}_{\mathrm{Se}}$ is located near the VBM. For SnSe, the VBM of perfect state is mainly contributed by the $\mathrm{Sn} 5 s$ and $\mathrm{Se} 4 p$ states, and the CBM is dominated by the $\mathrm{Sn} 5 p$ and Se $4 p$ states. As the $\mathrm{V}_{\mathrm{Sn}}$ is introduced, the metallicity is caused by the $\mathrm{Sn} 5 s$ and $\mathrm{Se} 4 p$ states distributing on the Fermi level. For defect $\mathrm{SnSe}$ with $\mathrm{V}_{\mathrm{Se}}$, the impurity level is also near the VBM, similar to the case of GeSe. The calculations show that in both GeSe and $\mathrm{SnSe}$, the $\mathrm{V}_{\mathrm{Se}}$ acts as a deep donor, and the effects of vacancy defects on the electronic structure of GeSe and SnSe are very similar to each other.

Effective mass characterizes band dispersion and is one of the important parameters to understand the electronic and transport properties of materials. To analyze the carrier transport, the effective masses of electrons and holes of ideal and defective SnSe and 
GeSe are calculated by the definition [52]: $m^{*}= \pm h\left(\frac{\partial^{2} E}{\partial k^{2}}\right)^{-1}$, where $E$ is the energy of band as a function of the wave vector $k$. The results are presented in Table 4 . It is notice that the electrons and holes effective mass in GeSe are always larger than those in $\mathrm{SnSe}$, and the values of electrons effective mass are always smaller than those of holes in both GeSe and SnSe compounds. For GeSe, the electrons effective mass decrease from $0.64 \mathrm{~m}_{0}$ to $0.54 \mathrm{~m}_{0}$ and holes effective mass increase from $0.66 \mathrm{~m}_{0}$ to $0.93 \mathrm{~m}_{0}$ with the introduction of $\mathrm{V}_{\mathrm{Ge}}$. For the case of $\mathrm{SnSe}$, the electrons effective mass of $0.28 \mathrm{~m}_{0}$ and holes effective mass of $0.42 \mathrm{~m}_{0}$ for SnSe with $\mathrm{V}_{\mathrm{Sn}}$ are closer to that of 0.25 and $0.45 \mathrm{~m}_{0}$ for perfect crystal, respectively, indicating that the influences of $\mathrm{V}_{\mathrm{Sn}}$ on the electron and hole effective mass are more slightly. For the introduction of Se vacancy, the electrons effective mass of $2.02 \mathrm{~m}_{0}$ and holes effective mass of $2.78 \mathrm{~m}_{0}$ for defective crystal are much larger than those of perfect crystal in GeSe. Similarly, the effective mass of $0.47 \mathrm{~m}_{0}$ and $1.43 \mathrm{~m}_{0}$ for electrons and holes in defective crystal are also larger than those of perfect crystal in SnSe. These results indicate that the introduction of anions vacancies result in the lower carrier mobility and reduced performance of radiation detection due to the introduction of impurity level near VBM arising from the existence of vacancy.

\section{Conclusions}

In summary, the formation energy, migration energy barrier and migration pathway for vacancy defects in GeSe and $\mathrm{SnSe}$ are systematically studied using a firstprinciples method. These results show that in GeSe the $\mathrm{V}_{\mathrm{Ge}}$ is more favorable than $\mathrm{V}_{\mathrm{Se}}$ 
under both Ge-rich and Se-rich conditions, and the vacancy formation in SnSe depends on the chemical environment, i.e., the $\mathrm{V}_{\mathrm{Se}}$ is more preferable under Sn-rich condition while the $\mathrm{V}_{\mathrm{Sn}}$ is easier to form under Se-rich condition. Among different diffusion paths, the [322] direction is shown to be the most favorable. The migration energy barriers along the [322] direction are predicted to be $0.54 \mathrm{eV}$ for $\mathrm{V}_{\mathrm{Ge}}$ in $\mathrm{GeSe}$ and 0.46 $\sim 0.52 \mathrm{eV}$ for $\mathrm{V}_{\mathrm{Sn}}$ in $\mathrm{SnSe}$, indicating that both vacancies are relatively easy to migrate in their respective compounds, which may result in the formation of vacancy clusters in $\mathrm{GeSe}$ and SnSe crystals. Besides, it is found that the cation vacancies are easier to migrate than anion vacancies in GeSe and SnSe. Generally, the vacancy formation and migration in GeSe and SnSe exhibit similar character, and the migration of $\mathrm{V}_{\mathrm{Sn}}$ is slightly much easier than that of $\mathrm{V}_{\mathrm{Ge}}$ due to the weaker interaction of $<\mathrm{Sn}$-Se $>$ bonding than that of $<\mathrm{Ge}-\mathrm{Se}>$ bonding. In addition, introduction of vacancy defect in GeSe and SnSe modifies their electronic structures significantly, i.e., band gap is reduced or metallicity is induced. The existence of cations vacancies effect slightly on the transport properties and interdiction of anions vacancies results in lower mobility and reduced transport properties of GeSe and SnSe. Fortunately, the anions vacancies are difficult to formation and migration in GeSe and SnSe. It predicts that the GeSe and SnSe are promising material for application of nuclear detector devices.

\section{Acknowledgements}

H.Y. Xiao was supported by the Joint funding of National Natural Science Foundation of China (Grant No. U1930120). X.T. Zu supported by the NSAF Joint 
Foundation of China (Grant No. U1630126). The theoretical calculations were performed using the supercomputer resources at TianHe-1 located at National Supercomputer Center in Tianjin. 


\section{Reference:}

[1] Shi G and Kioupakis E 2015 Nano Lett. 156926

[2] Antunez P D, Buckley J J and Brutchey R L 2011 Nanoscale 32399

[3] Dewandre A, Hellman O, Bhattacharya S, Romero A H, Madsen G K H and Verstraete M J 2016 Phys. Rev. Lett. 117276601

[4] Koski K J and Cui Y 2013 Acs Nano 73739

[5] Mao Y L, Xu C S, Yuan J M and Zhao H Q 2019 J Mater. Chem. A 711265

[6] Hsueh H C, Vass H, Clark S J, Ackland G J and Crain J 1995 Phy. Rev. B 5116750

[7] Yu L M, Degiovanni A, Thiry P A, Ghijsen J and Caudano R 1993 Phy. Rev. B 4716222

[8] Razykova T M et al 2018 Sol. Energy 159834

[9] Chen B et al 2019 Nanoscale 113968

[10] Yang S G, Wu M G, Wang B, Zhao L-D, Huang L, Jiang C and Wei S-H 2017 ACS Appl. Mater. Inter. 942149

[11] Yu W Y, Zhu Z L, Zhang S L, Cai X L, Wang X F, Niu C Y and Zhang W B 2016 Appl. Phys. Lett. 109172

[12] Zhao L D, Chang C, Tan G J and Kanatzidis M G 2016 Energ. Environ. Sci. 93044

[13] Yap W C, Yang Z, Mehboudi M, Yan J-A, Barraza-Lopez S and Zhu W 2018 Nano. Res. 11420

[14] Shi W, Gao M, Wei J, Gao J, Fan C, Ashalley E, Li H and Wang Z 2018 Adv. Sci. 51700602

[15] Im H S, Lim Y R, Cho Y J, Park J, Cha E H and Kang H S 2014 J. Phys. Chem. C 11821884

[16] Zhang L, Lu L, Zhang D, Hu W, Wang N, Xu B, Li Y and Zeng H 2016 Electrochim. Acta 209423

[17] Ramasamy P, Kwak D, Lim D H, Ra H S and Lee J S 2016 J Mater. Chem. C 4479

[18] Mukherjee B, Cai Y, Tan H R, Feng Y P, Tok E S and Sow C H 2013 ACS Appl. Mater. Inter. 59594

[19] Zheng D et al 2018 Acs Nano 127239

[20] Yao J, Zheng Z and Yang G 2017 Adv. Funct. Mater. 271701823

[21] Zhou X, Hu X, Jin B, Yu J, Liu K, Li H and Zhai T 2018 Adv. Sci. 51800478

[22] Bolotnikov A E et al 2009 Ieee T. Nucl. Sci. 561775

[23] Donegani E M, Fretwurst E, Garutti E, Klanner R, Lindstroem G, Pintilie I, Radu R and Schwandt J 2018 Nucl. Ins.Trum. Meth. A 89815

[24] Pintilie I, Lindstroem G, Junkes A and Fretwurst E 2009 Nucl. Ins.Trum. Meth. A 61152

[25] Dzhakhangirli Z A 2011 Russ. Phys. J+. 54277

[26] Jahangirli Z 2011 Turk. J. Phys. 35203

[27] Gomes L C, Carvalho A and Neto A H C 2016 Phy. Rev. B 94054103

[28] Zhou Y, Li W, Wu M, Zhao L-D, He J, Wei S-H and Huang L 2018 Phy. Rev. B 97245202

[29] Huang Y, Wang C, Chen X, Zhou D, Du J, Wang S and Ning L 2017 Rsc. Adv. 727612

[30] Sraitrova K, Cizek J, Holy V, Plechacek T, Benes L, Jarosova M, Kucek V and Drasar C 2019 Phy. Rev. B 99035306

[31] Kresse G and Hafner J 1993 Phy. Rev. B 47558

[32] Payne M C, Teter M P, Allan D C, Arias T A and Joannopoulos J D 1992 Rev. Mod. Phys. 641045

[33] Perdew J P, Burke K and Ernzerhof M 1996 Phys. Rev. Lett. 773865

[34] Zhou Y G 2016 J Mater. Chem. A 410906

[35] Xiao H Y and Weber W J 2011 J Phys. Chem. B 1156524

[36] Makinistian L and Albanesi E A 2011 Comp. Mater. Sci. 502872

[37] Song H Y and Lu J T 2018 Chemical Physics Letters 695200

[38] Okazaki A 1958 J Phys. Soc. Jpn. 131151 
[39] Ding G, Gao G and Yao K 2015 Sci. Rep. 59567

[40] Khan A A, Khan I, Ahmad I and Ali Z 2016 Mat. Sci. Semicon. Proc. 4885

[41] Gomes L C and Carvalho A 2015 Phy. Rev. B 92085406

[42] Lefebvre I, Szymanski M A, Olivier-Fourcade J and Jumas J C 1998 Phy. Rev. B 581896

[43] Kumagai Y and Oba F 2014 Phy. Rev. B 89195205

[44] Van de Walle C G and Neugebauer J 2004 J Appl. Phys. 953851

[45] Jiang M, Xiao H Y, Peng S M, Qiao L, Yang G X, Liu Z J and Zu X T 2018 Nanoscale Res. Lett. 13301

[46] Shannon R D 1976 Acta Cryst. A 32751

[47] Kang J S, Wu H, Li M and Hu Y J 2019 Nano Lett. 194941

[48] Deringer V L, Stoffel R P and Dronskowski R 2014 Phy. Rev. B 89094303

[49] Wiedemeier H and Siemers P A 1975 Z. Anorg. Allg. Chem. 41190

[50] Sorkina A and Adams S 2020 Materials Advances 1184

[51] Kyrtsos A, Matsubara M and Bellotti E 2017 Phy. Rev. B 95245202

[52] Du C Y, Tian J Y and Liu X J 2020 Materials Chemistry and Physics 249122961

[53] Shaabani L, Aminorroaya-Yamini S, Byrnes J, Nezhad A A and Blake G R 2017 Acs Omega 29192

[54] Walsh A and Watson G W 2005 J Phys. Chem. B 10918868 
Table 1. The calculated lattice constants of GeSe and SnSe. The experimental and theoretical results available in the literature are listed for comparison.

\begin{tabular}{ccccc}
\hline Compound & & $\mathrm{a}(\AA)$ & $\mathrm{b}(\AA)$ & $\mathrm{c}(\AA)$ \\
\hline \multirow{2}{*}{ GeSe } & Our Cal. & $\mathbf{1 1 . 1 2}$ & $\mathbf{3 . 8 7}$ & $\mathbf{4 . 5 5}$ \\
& Exp.[38] & 10.79 & 3.82 & 4.38 \\
& Exp.[53] & 10.84 & 3.84 & 4.40 \\
& Other Cal.[39] & 10.95 & 3.83 & 4.49 \\
& Other Cal.[40] & 10.85 & 3.9 & 4.331 \\
& Other Cal.[41] & 11.31 & 3.91 & 4.45 \\
& Our Cal. & $\mathbf{1 1 . 7 4}$ & $\mathbf{4 . 2 1}$ & 4.54 \\
& Exp.[42] & 11.50 & 4.15 & 4.44 \\
& Other Cal.[39] & 11.75 & 4.20 & 4.44 \\
& Other Cal.[40] & 11.53 & 4.17 & 4.4 \\
& Other Cal.[41] & 11.81 & 4.22 & 4.47 \\
& Other Cal.[54] & 11.74 & 4.21 & 4.57 \\
\hline
\end{tabular}


Table 2. The vacancy defect formation energy $(\mathrm{eV})$ of $\mathrm{XSe}(\mathrm{X}=\mathrm{Ge}$ or $\mathrm{Se})$ under $\mathrm{X}$-rich and Se-rich conditions. The theoretical results available in the literature are also listed for comparison. $\mathrm{V}_{\mathrm{x}}$ : $\mathrm{X}$ vacancy $(\mathrm{X}=\mathrm{Ge}$ or $\mathrm{Sn})$.

\begin{tabular}{cccccc}
\hline & & \multicolumn{2}{c}{$\mathrm{GeSe}$} & \multicolumn{2}{c}{$\mathrm{SnSe}$} \\
& & $\mathrm{V}_{\mathrm{Ge}}$ & $\mathrm{V}_{\mathrm{Se}}$ & $\mathrm{V}_{\mathrm{Sn}}$ & $\mathrm{V}_{\mathrm{Se}}$ \\
\hline Our Cal. & X-rich & 1.33 & 1.59 & 1.74 & 1.23 \\
& Se-rich & 0.86 & 2.06 & 0.84 & 2.02 \\
Other Cal. & X-rich & $1.14^{\mathrm{a}}$ & $1.59^{\mathrm{a}}$ & $1.78^{\mathrm{b}}$ & $1.17^{\mathrm{b}}$ \\
& Se-rich & $0.68^{\mathrm{a}}$ & $2.05^{\mathrm{a}}$ & $0.93^{\mathrm{b}}$ & $2.03^{\mathrm{b}}$ \\
\hline
\end{tabular}

${ }^{\mathrm{a} R e f . ~[18] ; ~}{ }^{\mathrm{b}} \operatorname{Ref}$ [28]. 
Table 3 The $<\mathrm{X}-\mathrm{Se}>$ bonding distances $(\AA)$ in defective XSe $(\mathrm{X}=\mathrm{Ge}$ or $\mathrm{Sn})$. The $\Delta d$ represents the distance difference between $<\mathrm{Ge}-\mathrm{Se}>$ and $<\mathrm{Sn}-\mathrm{Se}>$ bonds. $\mathrm{V}_{\mathrm{x}}$ : $\mathrm{X}$ vacancy $(\mathrm{X}=\mathrm{Ge}$ or $\mathrm{Sn})$.

\begin{tabular}{ccccc}
\hline Sites & Bonds & $\mathrm{V}_{\mathrm{Ge}}$ & $\mathrm{V}_{\mathrm{Sn}}$ & $\Delta d(\AA)$ \\
\hline Saddle-point A & $<\mathrm{X}-\mathrm{Se} 1>$ & 2.34 & 2.53 & 0.19 \\
& $<\mathrm{X}-\mathrm{Se} 2>$ & 2.51 & 2.61 & 0.1 \\
& $<\mathrm{X}-\mathrm{Se} 3>$ & 2.48 & 2.66 & 0.18 \\
& $<\mathrm{X}-\mathrm{Se} 4>$ & - & - & - \\
Local minia site & $<\mathrm{X}-\mathrm{Se} 1>$ & 2.36 & 2.54 & 0.18 \\
& $<\mathrm{X}-\mathrm{Se} 2>$ & 2.42 & 2.56 & 0.14 \\
& $<\mathrm{X}-\mathrm{Se} 3>$ & 2.53 & 2.70 & 0.17 \\
& $<\mathrm{X}-\mathrm{Se} 4>$ & 2.49 & 2.68 & 0.19 \\
& $<\mathrm{X}-\mathrm{Se} 1>$ & 2.30 & 2.61 & 0.31 \\
& $<\mathrm{X}-\mathrm{Se} 2>$ & 2.36 & 2.57 & 0.21 \\
& $<\mathrm{X}-\mathrm{Se} 3>$ & - & - & - \\
& $<\mathrm{X}-\mathrm{Se} 4>$ & 2.38 & 2.66 & 0.28 \\
\hline
\end{tabular}


Table 4 The effective mass of electrons $\left(m_{e}\right)$ and holes $\left(m_{h}\right)$ for perfect and defective GeSe and SnSe, $V_{X}$ : X vacancy (X=Ge, Sn or Se).

\begin{tabular}{cccc}
\hline Crystal & & $\mathrm{m}_{\mathrm{e}}\left(\mathrm{m}_{0}\right)$ & $\mathrm{m}_{\mathrm{h}}\left(\mathrm{m}_{0}\right)$ \\
\hline \multirow{2}{*}{ GeSe } & Perfect & 0.64 & 0.66 \\
& $\mathrm{~V}_{\mathrm{Ge}}$ & 0.54 & 0.93 \\
& $\mathrm{~V}_{\mathrm{Se}}$ & 2.02 & 2.78 \\
\multirow{2}{*}{$\mathrm{SnSe}$} & Perfect & 0.25 & 0.45 \\
& $\mathrm{~V}_{\mathrm{Sn}}$ & 0.28 & 0.42 \\
& $\mathrm{~V}_{\mathrm{Se}}$ & 0.47 & 1.43 \\
\hline
\end{tabular}


Figure caption

Figure 1 A schematic view of geometrical structure of unit cell for $\mathrm{XSe}(\mathrm{X}=\mathrm{Ge}, \mathrm{Sn})$.

The considered migration directions for $\mathrm{V}_{\mathrm{X}}(\mathrm{X}=\mathrm{Ge}, \mathrm{Sn})$ and $\mathrm{V}_{\mathrm{Se}}$ are indicated by the dashed arrow. $V_{X}$ : X vacancy $(X=G e, S n), V_{\text {Se: }}$ Se vacancy. The black and blue dashed lines with arrow indicate $\mathrm{V}_{\mathrm{X}}$ and $\mathrm{V}_{\text {se }}$ migration direction in the same layer, and the red dashed line with arrow indicates the vacancy migration direction between two neighboring layers.

Figure 2 The energy change with diffusion coordinate for Ge vacancy migration along [010], [322], [001] and [011] directions in GeSe.

Figure 3 The energy change with diffusion coordinate for Sn vacancy migration along [010], [322], [001] and [011] directions in SnSe.

Figure 4 A schematic view of optimized local configurations for saddle-points and local minima site during (a) $\mathrm{V}_{\mathrm{Ge}}$ migration in GeSe and (b) $\mathrm{V}_{\mathrm{Sn}}$ migration in $\mathrm{SnSe}$ along the [322] direction. $\mathrm{V}_{\mathrm{x}}$ : $\mathrm{X}$ vacancy $(\mathrm{X}=\mathrm{Ge}$ or $\mathrm{Sn})$.

Figure 5 The energy change with diffusion coordinate for Se vacancy migration along [010], $[\overline{5} \overline{4} \overline{4}],[001]$ and [011] directions in GeSe.

Figure 6 The energy change with diffusion coordinate for Se vacancy migration along [010], $[\overline{5} \overline{4} \overline{4}],[001]$ and [011] directions in SnSe.

Figure 7 The energy change with diffusion coordinate for the migration of $\mathrm{Ge}$ and $\mathrm{Se}$ vacancy in GeSe and Sn and Se vacancy in SnSe between two neighboring layers. Figure 8 Variation of migration energy barrier with temperature for $V_{G e}$ and $V_{S n}$ migration along the [322] direction. 
Figure 9 The band structure of perfect (a) GeSe and (d) SnSe, defective GeSe with (b) $\mathrm{V}_{\mathrm{Ge}}$ and (c) $\mathrm{V}_{\mathrm{Se}}$, and defective SnSe with (e) $\mathrm{V}_{\mathrm{Sn}}$ and (f) $\mathrm{V}_{\mathrm{Se}} . \mathrm{V}_{\mathrm{x}}$ : $\mathrm{X}$ vacancy (X=Ge, Sn or Se). (g) Schematic view of the first Brillouin zone and the K-path for band structure calculations in reciprocal space for orthorhombic structure of GeSe and SnSe. All K-path are considered to calculate the band structure, and we only show the the band structure with path G-X-S-Y-G-Z, which already is including the VBM and CBM.

Figure 10 The projected density of states for perfect and defective (a) GeSe and (b) $\mathrm{SnSe}, \mathrm{V}_{\mathrm{x}}: \mathrm{X}$ vacancy $(\mathrm{X}=\mathrm{Ge}, \mathrm{Sn}$ or $\mathrm{Se})$. The fermi level is set to be $0 \mathrm{eV}$. 


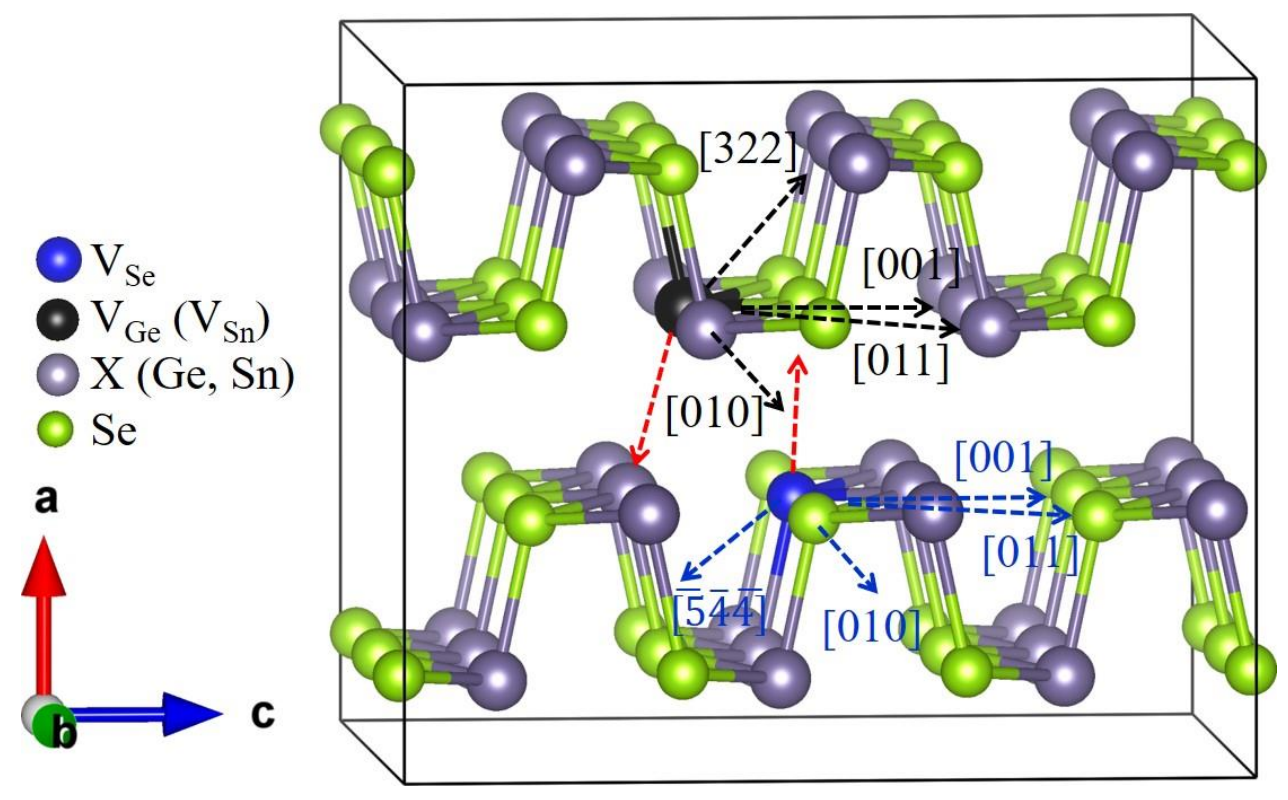

Figure 1 
(a) Ge-V
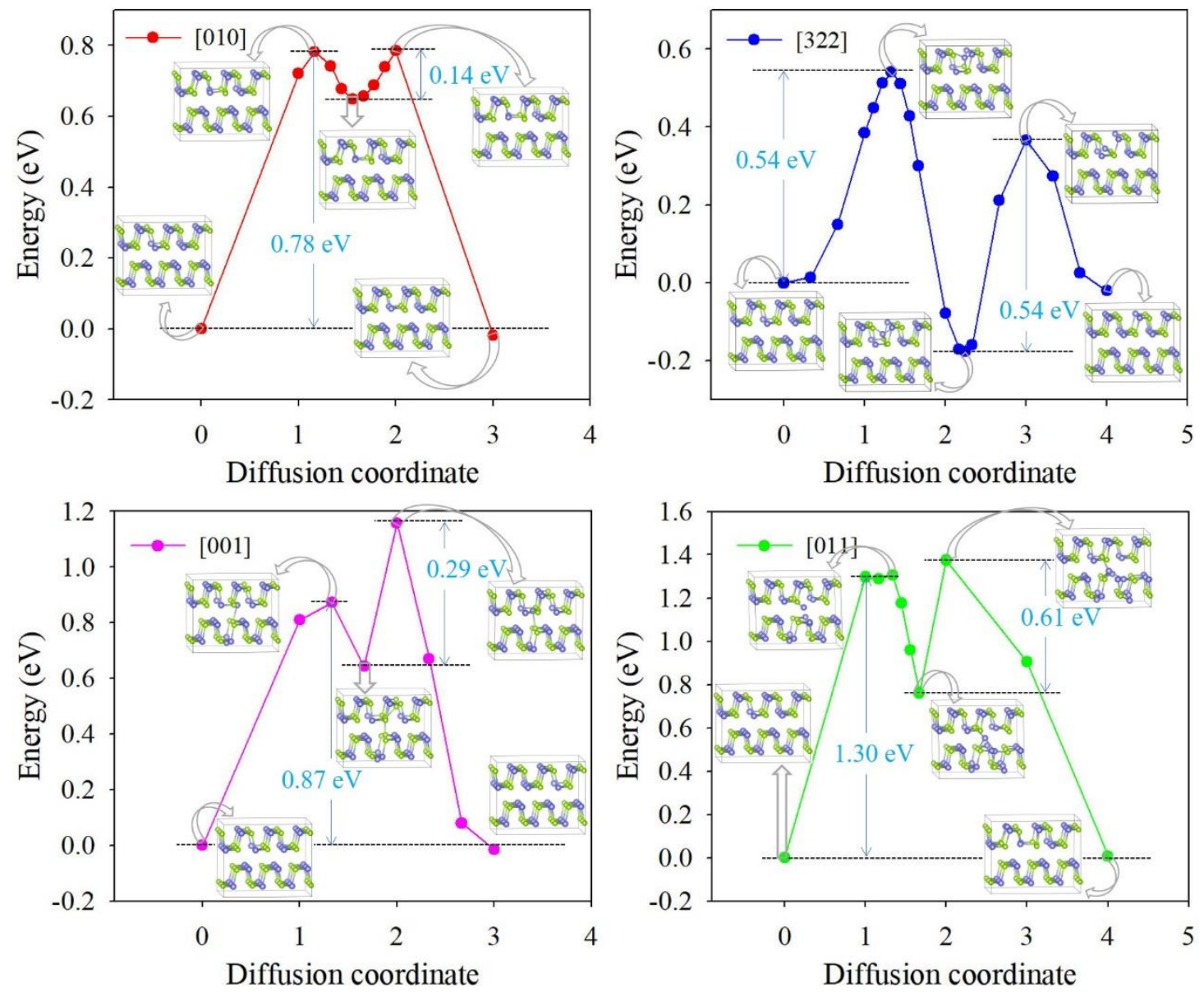

Figure 2 
(b) Sn-V
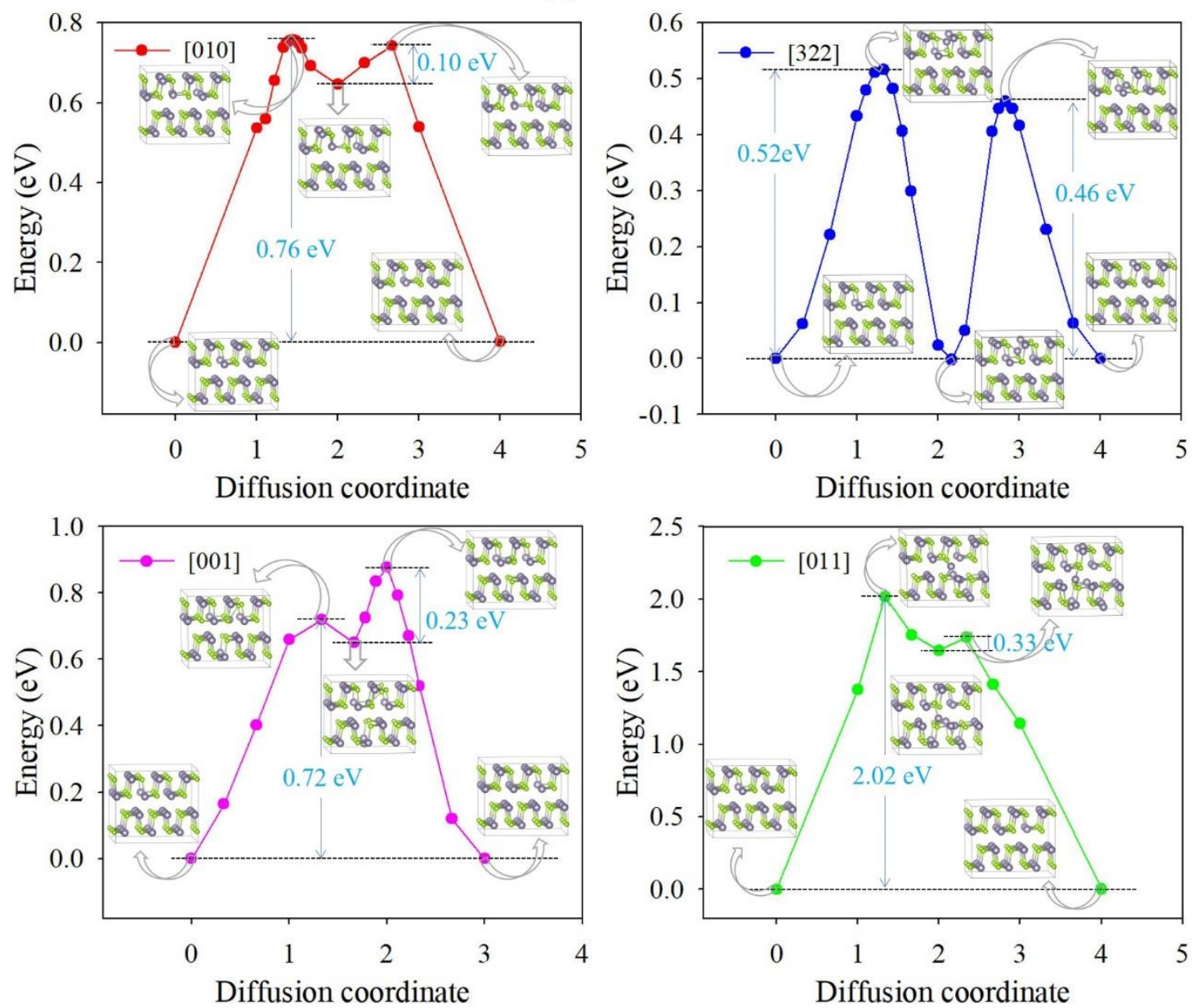

Figure 3 
(a) $\mathrm{V}_{\mathrm{Ge}}$

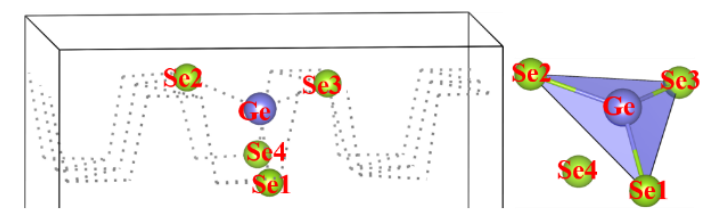

Saddle-point A

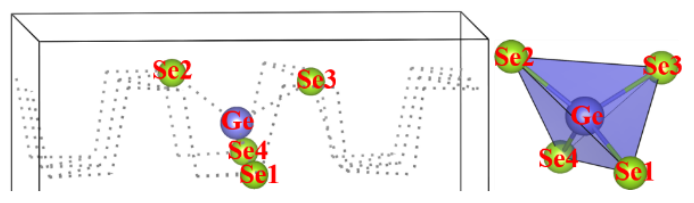

Local minimum site

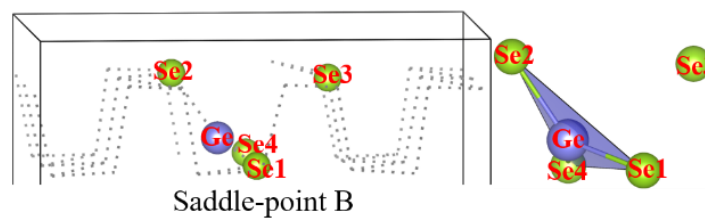

Saddle-point B (b) $\mathrm{V}_{\mathrm{Sn}}$

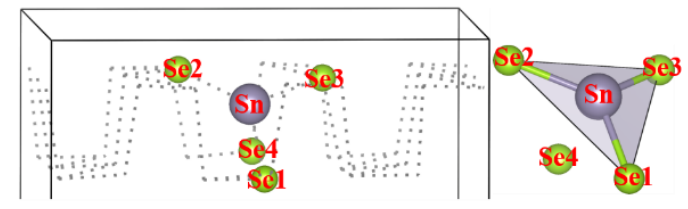

Saddle-point A

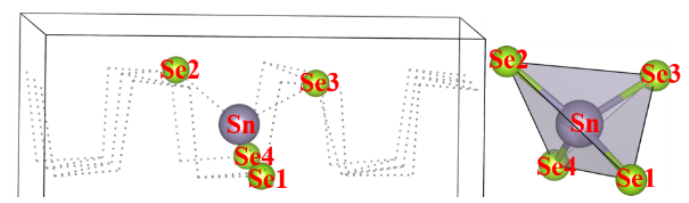

Local minimum site

3

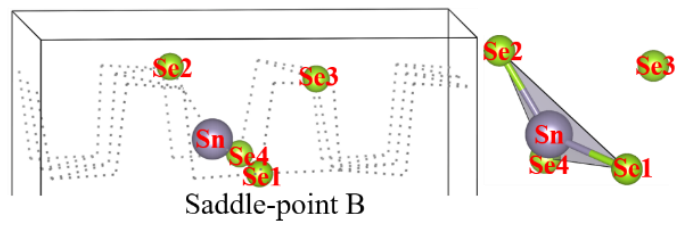

Figure 4 
(a) $\mathrm{V}_{\mathrm{Se}}$ in $\mathrm{GeSe}$
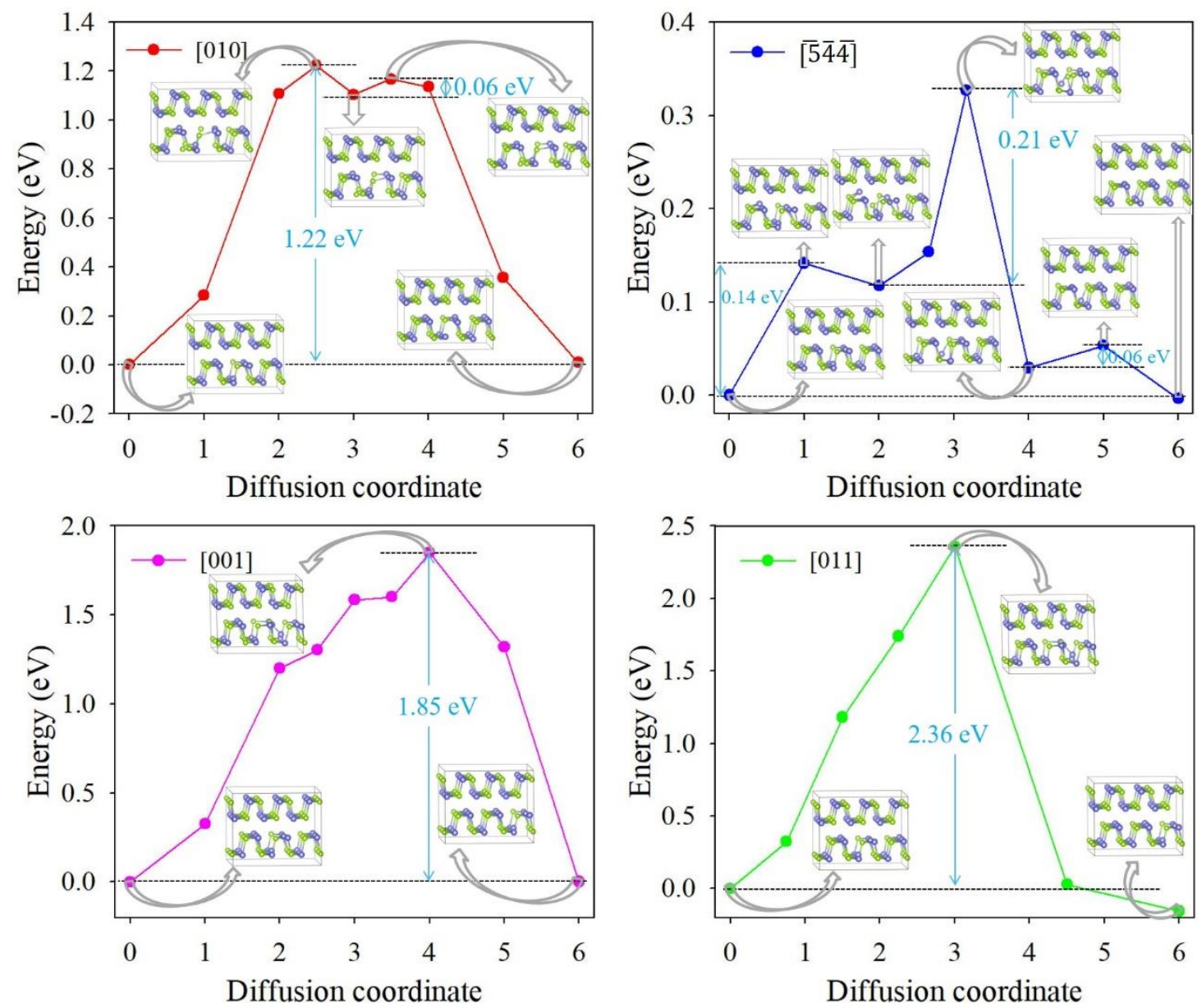

Figure 5 
(a) $\mathrm{V}_{\mathrm{Se}}$ in $\mathrm{SnSe}$
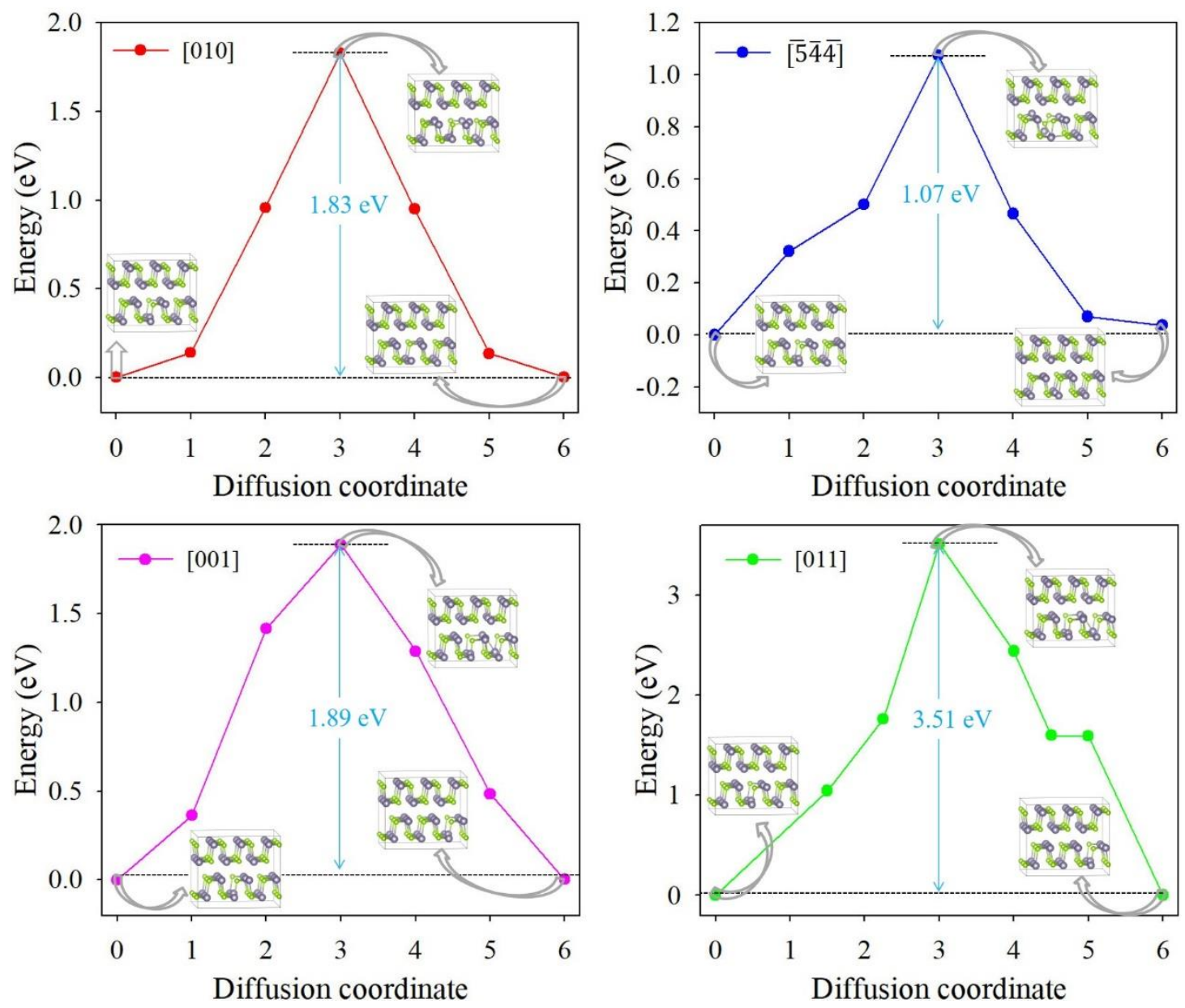

Figure 6 

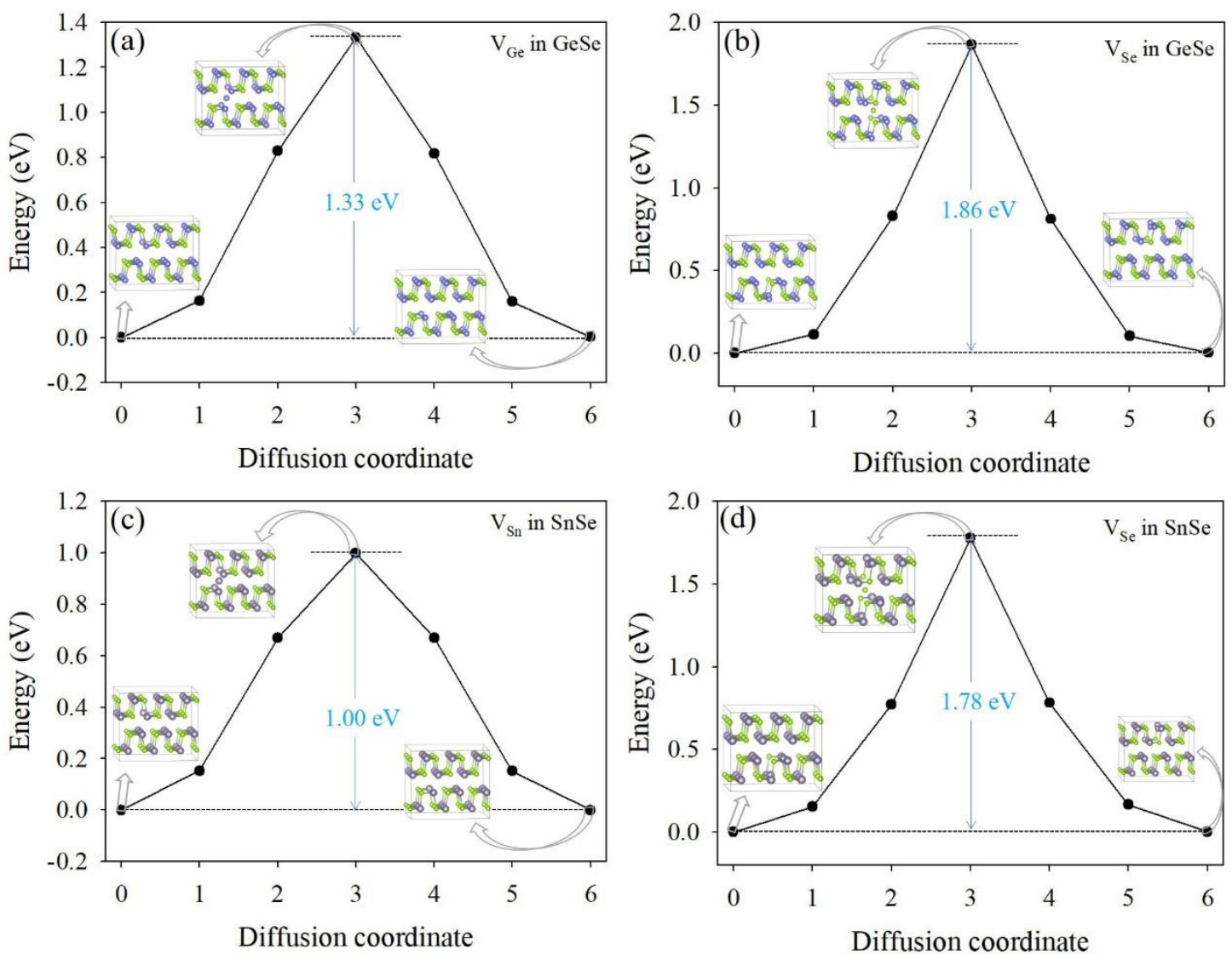

Figure 7 


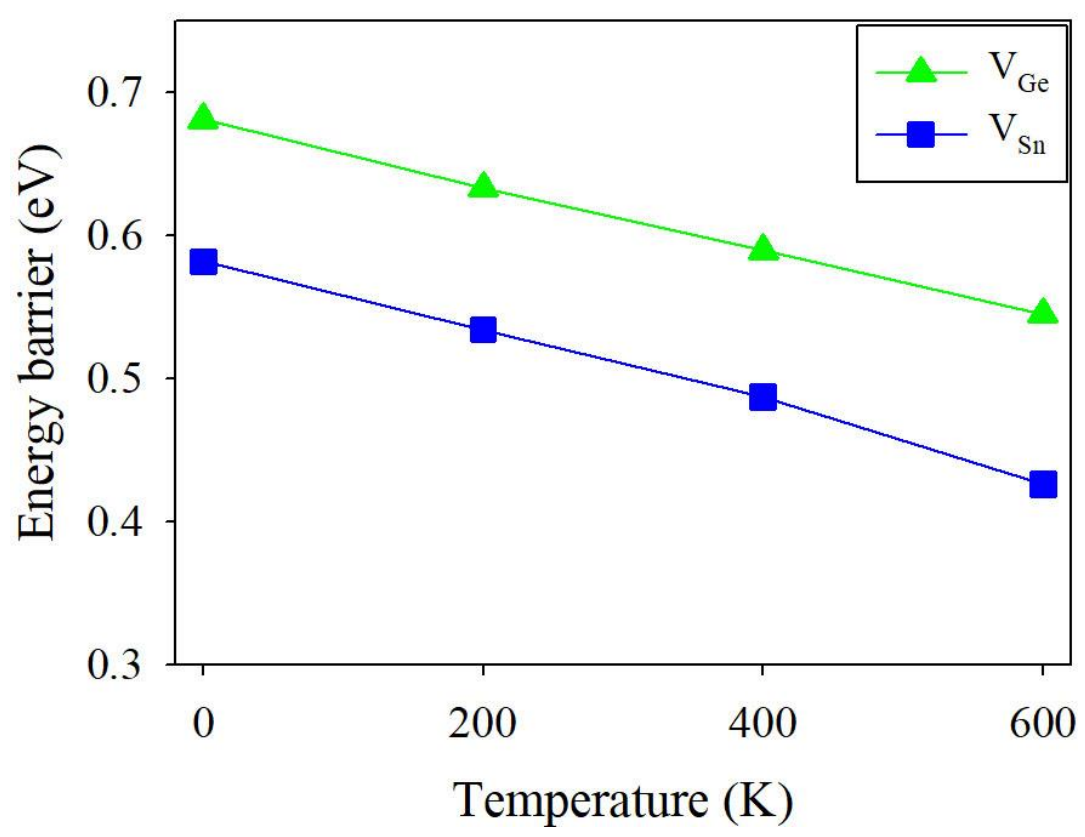

Figure 8 
(a) Perfect 4

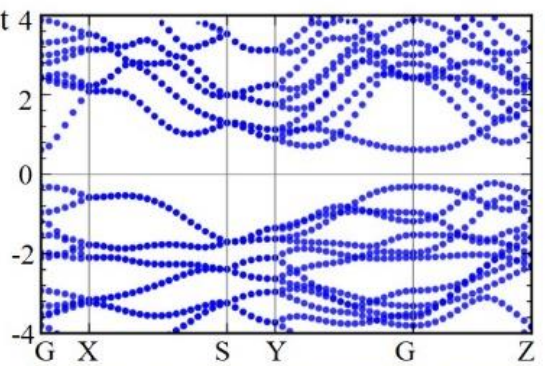

(b) $\mathrm{V}_{\mathrm{Ge}}$

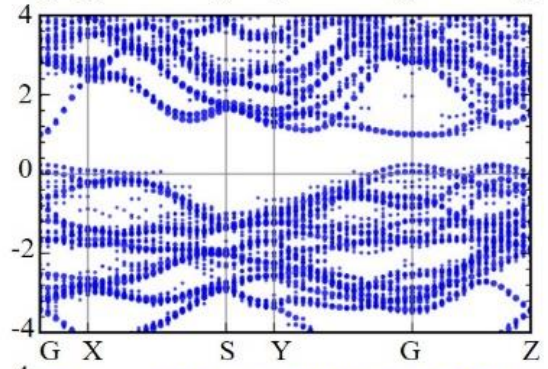

(c) $\mathrm{V}_{\mathrm{Se}}$

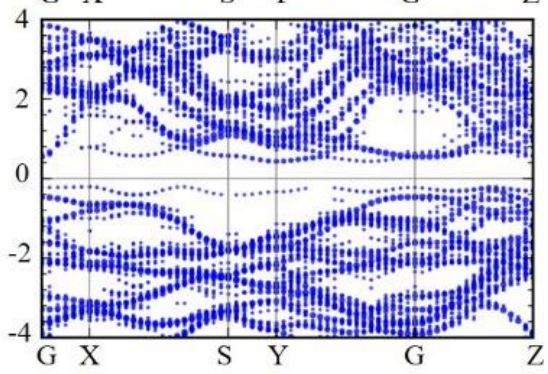

(g)

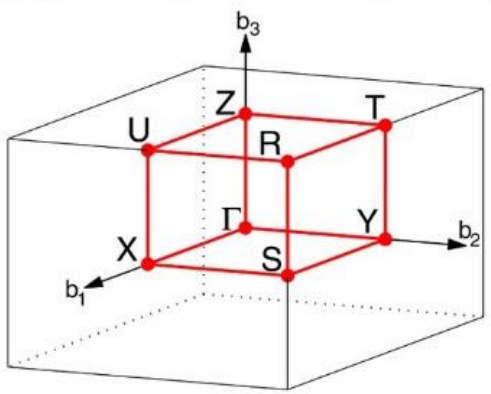

(d) Perfect 4

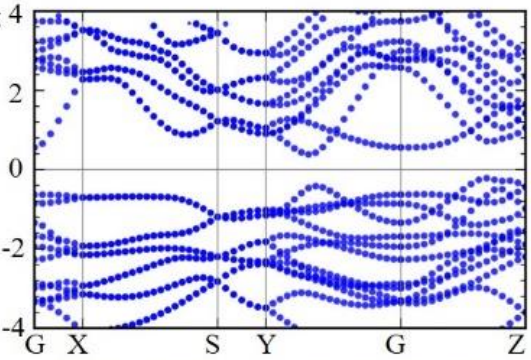

(e) $V_{S n}$

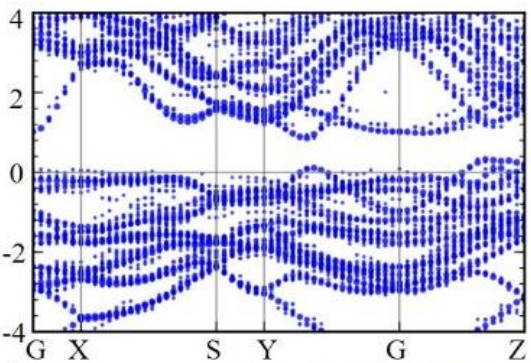

(f) $\mathrm{V}_{\mathrm{Se}}$

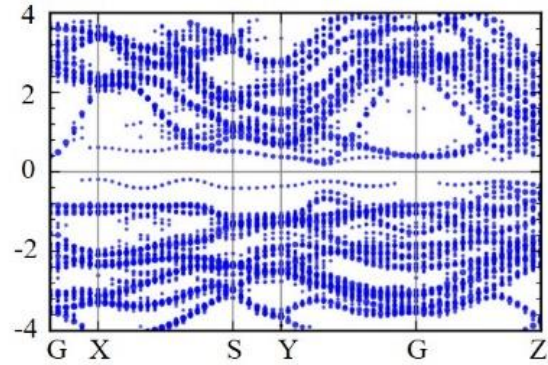

Figure 9 
(a)

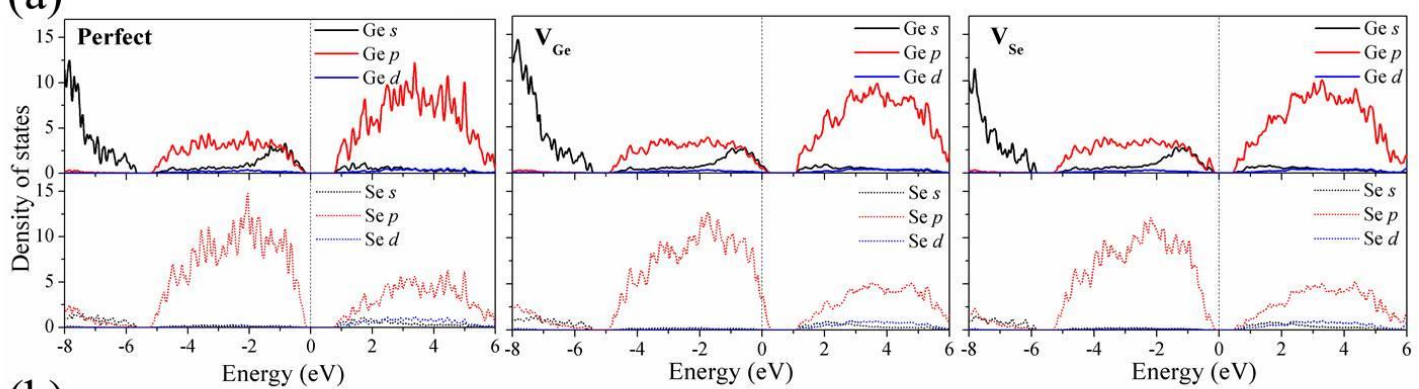

(b)

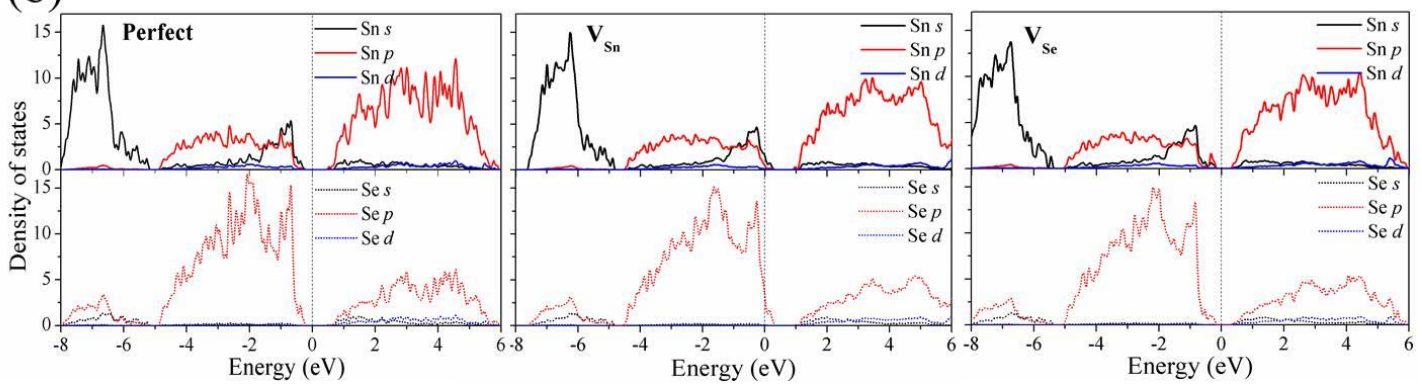

Figure 10 